OPEN

SUBJECT AREAS:

HIV INFECTIONS

VIRAL TRANSMISSION

VIRAL EVOLUTION

VIRAL IMMUNE EVASION

Received

3 May 2013

Accepted

6 May 2014

Published

28 May 2014

Correspondence and requests for materials should be addressed to

N.A.K. (N.A.

Kootstra@amc.uva.nl)

* Current address:

Crucell Holland BV,

Leiden, the

Netherlands.

\section{Low level of HIV-1 evolution after transmission from mother to child}

\author{
Esther F. Gïsbers' ${ }^{1}$ Ad C. van Nuenen', Alba Torrents de la Peňa', Emma J. Bowles², \\ Guillaume B. Stewart-Jones ${ }^{2}$, Hanneke Schuitemaker ${ }^{1 *}$ \& Neeltje A. Kootstra'
}

\begin{abstract}
'Department of Experimental Immunology, Sanquin Research, Landsteiner Laboratory, and Center for Infectious Diseases and Immunity Amsterdam (CINIMA), Academic Medical Center, University of Amsterdam, Amsterdam, the Netherlands, ${ }^{2}$ Human Immunology Unit, Weatherall Institute of Molecular Medicine, John Radcliffe Hospital, University of Oxford, Oxford, United Kingdom.
\end{abstract}

Mother-to-child HIV-1 transmission pairs represent a good opportunity to study the dynamics of CTL escape and reversion after transmission in the light of shared and non-shared HLA-alleles. Mothers share half of their HLA alleles with their children, while the other half is inherited from the father and is generally discordant between mother and child. This implies that HIV-1 transmitted from mother to child enters a host environment to which it has already partially adapted. Here, we studied viral evolution and the dynamics of CTL escape mutations and reversion of these mutations after transmission in the context of shared and non-shared HLA alleles in viral variants obtained from five mother-to-child transmission pairs. Only limited HIV-1 evolution was observed in the children after mother-to-child transmission. Viral evolution was mainly driven by forward mutations located inside CTL epitopes restricted by HLA alleles inherited from the father, which may be indicative of CTL pressure.

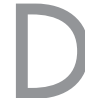

uring primary HIV-1 infection, the appearance of HIV-1 specific CTLs coincides with a decline in viral load and CTL responses contribute to control of viral replication ${ }^{1-7}$. HIV-1 can rapidly escape from CTLmediated immune pressure by mutations within or flanking the CTL epitopes that disrupt peptide binding to HLA molecules or T cell recognition of the epitope ${ }^{8-10}$, resulting in loss of control of viremia and disease progression ${ }^{11-13}$. Some CTL escape mutations, however, come at a fitness cost to the virus ${ }^{14-16}$, which is reflected in their reversion after transmission to a new host with discordant HLA alleles ${ }^{17,18}$.

Mothers share half of their HLA alleles with their children, while the other half is inherited from the father and is generally discordant between mother and child. This implies that HIV-1 transmitted from mother to child enters a host environment to which it has already partially adapted. During HIV-1 infection of the mother, maternal immune pressure drives selection of CTL escape variants. When these escape variants are transmitted, CTL recognition of epitopes restricted by HLA alleles that are shared between mother and child will be hampered in the child. However, as the infant's immune system has not yet developed, the transmitted escape mutations in the virus may rapidly revert due to a fitness disadvantage. The reverted epitopes can be presented and elicit an CTL response in the child, and subsequently viral escape variants may be selected again. Potential compensatory mutations restoring the loss of viral fitness associated with escape mutations in viral variants from the mother may be preserved after transmission to the child, resulting in the presence of CTL escape variants with optimal replication fitness early after transmission. This may contribute, at least in part, to the fast progression of pediatric HIV-1 infection ${ }^{19-21}$.

Transmitted virus variants with CTL escape mutations in epitopes restricted by non-shared HLA alleles have been described to revert to wild type sequence in some $\operatorname{cases}^{17,22,23}$, however persistence of these mutations has also been described ${ }^{24,25}$. Furthermore, viral epitopes presented by HLA alleles inherited from the father may be able to elicit CTL responses in the child and de novo HIV-specific CTL escape mutations can arise in the infant shortly after birth ${ }^{20,22,26,27}$.

Mother-to-child transmission pairs represent an opportunity to study the dynamics of CTL escape and reversion after transmission in the light of CTL epitopes restricted by shared and non-shared HLA alleles. Furthermore, viral evolution in children infected by their mother has not been studied extensively and mainly focused on escape in CTL epitopes restricted by protective HLA alleles, or in well characterized CTL epitopes in the most common HLA alleles. To gain a better understanding of viral evolution and the dynamics of forward mutations and reversion of transmitted mutations after transmission, we studied 5 mother-to-child transmission 
pairs and sequenced nef, gag, and env of HIV-1 variants obtained from both mothers and their children at time points close to delivery or birth, respectively. Genetic divergence and the number of synonymous and non-synonymous mutations were analyzed between viral variants obtained from mother and child, or from two successive time points from the child. The number of forward mutations and reversion of mutations in the viruses from the child were studied in the context of epitopes restricted by HLA alleles inherited from the mother or the father.

\section{Results}

HIV-1 transmission from mother to child. Five mother-child pairs with documented vertical HIV-1 transmission were included and $n e f, g a g$ and env sequences were generated from clonal HIV-1 variants (Genbank accession numbers KJ669727-KJ670070). Patient characteristics and the number of viral sequences analyzed are summarized in Table 1. One of the mothers (M114) transmitted HIV -1 to her child during pregnancy, as could be concluded on the basis of the presence of virus in umbilical cord blood samples, where contamination with maternal blood could be ruled out ${ }^{28,29}$. For the other children the exact moment of transmission was unknown.

Neighbour joining (NJ) phylogenetic analyses showed pair wise clustering of the nef, gag and env sequences from mothers and their children with high bootstrap values. Four pairs were infected with HIV-1 subtype B viruses, whereas mother-child pair 127 was infected with HIV-1 circulating recombinant form AG. Transmission of multiple viral variants was observed for pair 137 , with viral sequences from the child intermingled within viral sequences obtained from the mother. This was supported by sequences from all three viral genes under study. In the other four mother-child pairs, the viral sequences from the children formed monophyletic clusters, indicating outgrowth of one viral variant (supplementary figure 1).

Evolution of virus variants after transmission from mother to child. To assess the evolutionary rate of HIV-1 after transmission, we analyzed the genetic divergence of viral isolates close to childbirth in mothers and children for pairs 102, 127 and 137 (Table 2). For pair 114 only a limited number of viral variants was available for the mother and therefore, the genetic divergence was only analyzed for viral variants isolated at two successive time points from the child. From mother M133 only sequences from viral variants isolated after delivery were available, and we therefore analyzed the genetic divergence between sequences obtained from cord blood and 9.5 months after birth from the child (Table 2). Genetic distances between viral isolates obtained from the different pairs or from the children at different time points were low for gag (0.5-2\%) and nef (1.5-2.5\%) and somewhat higher for env (2-4\%).

To determine whether the genetic divergence observed was caused by selection pressure on the viruses, or whether the evolution was random due to the error-prone reverse transcriptase and high viral turn-over, we calculated the ratio of non-synonymous and synonymous mutations ( $\mathrm{dN} / \mathrm{dS}$ ratio). A dN/dS ratio smaller than 1 indicates negative selection pressure to retain protein function. A neutral dN/ $\mathrm{dS}$ ratio around 1 indicates an equal amount of synonymous and non-synonymous mutations and thus no specific selective pressure on the protein. When $\mathrm{dN} / \mathrm{dS}$ ratios are above 1 there is positive selection, indicating pressure on the protein to change amino acid composition to gain fitness or evade immune responses. When comparing nef sequences, mainly negative to neutral selection pressure was observed in viral variants obtained from children C102, C114 and C137, whereas viral sequences from children C127 and C133 showed positive selection. When analyzing gag viral sequences, we mainly observed negative selection in viral variants obtained from all pairs, with some positive selection observed for sequences obtained from child C114. In the gp160 of the envelope gene, negative to neutral selection was observed in viral isolates obtained from the different pairs and from the children at different time points. However, when comparing viral sequences encoding the variable regions of env positive selection was observed in the V1V2 region for children C133 and C137 and in the region V4 for children C102 and C127. Comparing viral sequences from child C114 isolated from two different time points showed slight positive selection in regions V1V2 and V4 (Table 2).

Reversions and forward mutations after transmission. As HIVspecific CTL responses are a major driving force for viral evolution after horizontal HIV-1 transmission and selection for CTL escape mutations occurs widely, we analyzed the number of reversions and forward mutations in viral sequences from the children in the light of predicted CTL epitopes restricted by HLA alleles carried by mothers and children. Forward mutations were defined as amino acid mutations in viral sequences in the child that were not observed in the viral sequences from the mother. Reversion of mutations in viruses from the children were defined as amino acid mutations present in virus variants from the mother that were transmitted to

Table 1 | Characteristics of mother-child transmission pairs

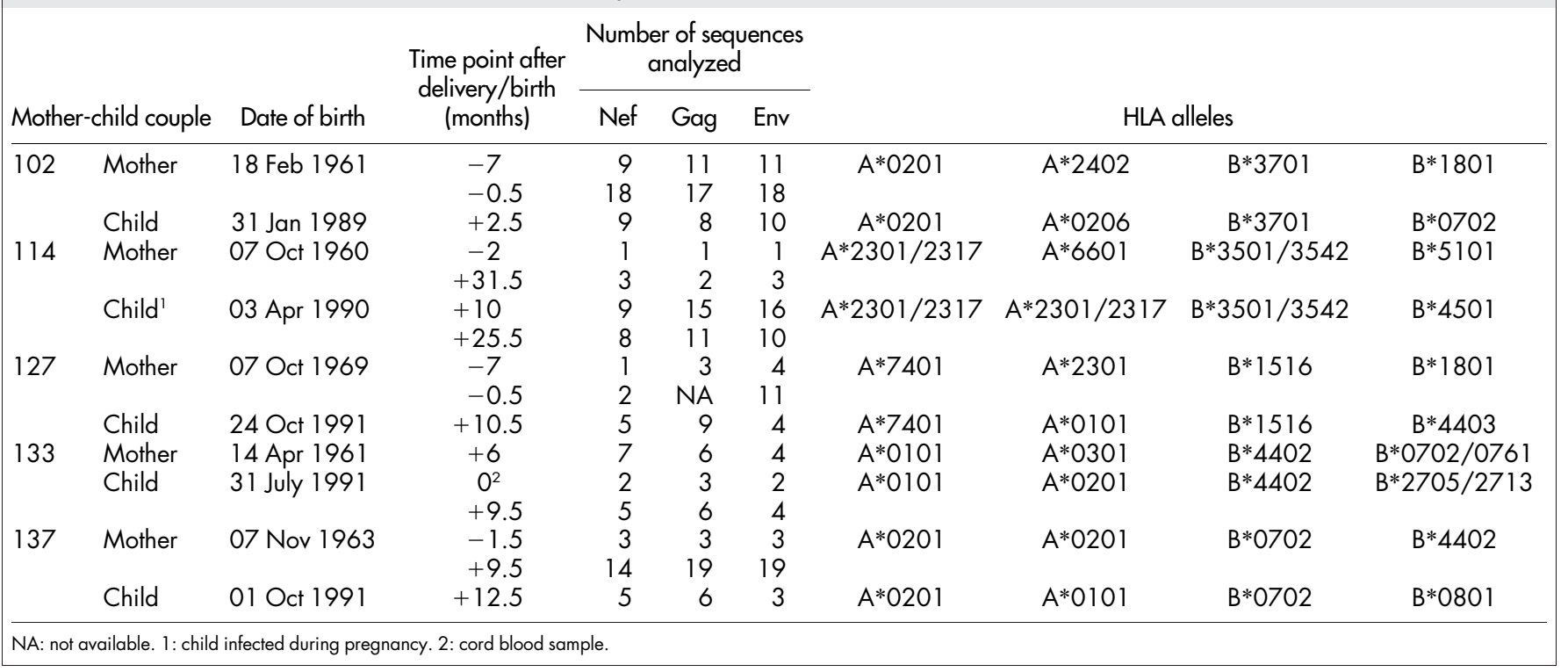




\begin{tabular}{|c|c|c|c|c|c|c|c|c|}
\hline \multirow[b]{2}{*}{ Mother-child couple } & \multirow{2}{*}{$\begin{array}{l}\text { Time point after delivery/ } \\
\text { birth (months) }\end{array}$} & \multirow[b]{2}{*}{ Gene } & \multicolumn{3}{|c|}{ Divergence } & \multicolumn{3}{|c|}{$d N / d S$} \\
\hline & & & Mean & SD & $95 \% \mathrm{Cl}^{1}$ & Mean & SD & $95 \% \mathrm{Cl}^{1}$ \\
\hline \multirow[t]{7}{*}{102} & Mother -0.5 & Nef & 1.42 & 0.38 & $0.66-2.18$ & 0.30 & 0.21 & $-0.12-0.71$ \\
\hline & Child +2.5 & Gag & 1.36 & 0.24 & $0.88-1.84$ & 0.14 & 0.11 & $-0.08-0.36$ \\
\hline & & Env & 2.67 & 0.2 & $2.27-3.07$ & 0.52 & 0.10 & $0.31-0.73$ \\
\hline & & V1V2 & & & & 0.70 & 0.33 & $0.04-1.37$ \\
\hline & & V3 & & & & 0.30 & 0.37 & $-0.45-1.04$ \\
\hline & & V4 & & & & 2.35 & 1.79 & $-1.23-5.94$ \\
\hline & & V5 & & & & 1.00 & 0.17 & $0.66-1.34$ \\
\hline \multirow[t]{7}{*}{114} & Child + 10 & Nef & 1.96 & 0.39 & $1.18-2.74$ & 0.73 & 0.53 & $-0.33-1.80$ \\
\hline & Child +25.5 & Gag & 0.59 & 0.11 & $0.37-0.81$ & 0.62 & 0.65 & $-0.68-1.91$ \\
\hline & & Env & 1.77 & 0.14 & $1.49-2.05$ & 0.51 & 0.14 & $0.24-0.79$ \\
\hline & & V1V2 & & & & 0.71 & 0.43 & $-0.12-1.57$ \\
\hline & & V3 & & & & 0.93 & 0.20 & $0.53-1.34$ \\
\hline & & V4 & & & & 0.71 & 0.39 & $-0.06-1.49$ \\
\hline & & V5 & & & & 0.82 & 0.86 & $-0.90-2.55$ \\
\hline \multirow[t]{7}{*}{127} & Mother -0.5 & Nef & 2.63 & 0.47 & $1.69-3.57$ & 1.98 & 1.62 & $-1.27-5.22$ \\
\hline & Child + 10.5 & $\mathrm{Gag}^{2}$ & 0.99 & 0.23 & $0.53-1.45$ & 0.22 & 0.08 & $0.06-0.39$ \\
\hline & & Env & 2.10 & 0.17 & $1.76-2.44$ & 0.73 & 0.21 & $0.31-1.14$ \\
\hline & & V1V2 & & & & 0.57 & 0.57 & $-0.58-1.71$ \\
\hline & & V3 & & & & 0.86 & 0.24 & $0.37-1.35$ \\
\hline & & V4 & & & & 2.00 & 1.09 & $-0.12-4.18$ \\
\hline & & V5 & & & & 0.84 & 0.27 & $0.30-1.38$ \\
\hline \multirow[t]{7}{*}{133} & Child $0^{3}$ & Nef & 1.86 & 0.42 & $1.02-2.70$ & 1.39 & 0.81 & $-0.22-3.01$ \\
\hline & Child +9.5 & Gag & 1.76 & 0.33 & $1.10-2.42$ & 0.30 & 0.08 & $0.15-0.45$ \\
\hline & & Env & 3.92 & 0.34 & $3.24-4.60$ & 0.66 & 0.06 & $0.54-0.79$ \\
\hline & & V1V2 & & & & 1.33 & 0.06 & $1.21-1.45$ \\
\hline & & V3 & & & & 0.62 & 0.42 & $-0.22-1.46$ \\
\hline & & V4 & & & & 1.01 & 0.24 & $0.53-1.50$ \\
\hline & & V5 & & & & 0.85 & 0.44 & $-0.04-1.74$ \\
\hline \multirow[t]{7}{*}{137} & Mother -1.5 & Nef & 1.41 & 0.35 & $0.71-2.11$ & 0.63 & 0.52 & $-0.42-1.68$ \\
\hline & Child + 12.5 & Gag & 1.11 & 0.22 & $0.67-1.55$ & 0.07 & 0.03 & $0.00-0.13$ \\
\hline & & Env & 2.35 & 0.23 & $1.89-2.81$ & 0.46 & 0.40 & $-0.34-1.26$ \\
\hline & & V1V2 & & & & 1.60 & 0.47 & $0.67-2.53$ \\
\hline & & V3 & & & & 0.36 & 0.16 & $0.04-0.68$ \\
\hline & & V4 & & & & 0.76 & 0.25 & $0.26-1.25$ \\
\hline & & V5 & & & & 0.76 & 0.36 & $0.04-1.48$ \\
\hline
\end{tabular}

the child and subsequent back mutation of this amino acid towards consensus sequence in viral variants from the child. The number of reversions and forward mutations were categorized as located inside CTL epitopes restricted by HLA alleles from the mother that are not shared with the child (maternal), HLA alleles that both mother and child carry (shared), or HLA alleles from the child that are not shared with the mother (paternal). The position and location within HLArestricted CTL epitopes of forward mutations and reversion of transmitted mutations in Nef, Gag and Env in viral variants obtained from the children are summarized in supplementary table 1.

Inside predicted CTL epitopes that were restricted by non-shared maternal HLA alleles, no reversion of transmitted mutations was observed in sequences from child 102. Only a low level of reversions was seen in sequences obtained from the other children, with only 2 reversions (12\%), taking place at an anchor residue of an CTL epitope restricted by non-shared HLA from the mother. The majority of reversions occurred in Env (Figure 1A). The number of forward mutations in epitopes restricted by non-shared HLA alleles was not determined, since these HLA alleles are not expressed in the children. These forward mutations are denoted as being located outside CTL epitopes.

When analyzing mutations occurring within CTL epitopes restricted by HLA alleles that are shared between mothers and children, a limited number of mutations were observed (Figure 1B). In 4 out of 5 children, the majority of these mutations were forward mutations. In viral variants from child 114 an equal number of reversions and forward mutations was observed. Approximately $18 \%$ of reversions and forward mutations were located at anchor positions of the CTL epitopes.

Evolution in CTL epitopes restricted by HLA alleles from the children that were inherited from the father was mainly driven by forward mutations in 4 out of 5 children (Figure 1C). In sequences obtained from child $\mathrm{C} 114$ more reversions than forward mutations were observed in these CTL epitopes. Approximately $30 \%$ of forward mutations were located at anchor residues of CTL epitopes restricted by HLA alleles from the children inherited from the father, whereas approximately $18 \%$ of reversions were located at anchor residues.

Viral evolution outside of predicted CTL epitopes was mainly driven by forward mutations, and the majority of mutations were observed in sequences from child C133 (Figure 1D).

Additionally, we compared the total number of reversions and forward mutations located in Nef, Gag and Env. After correction for the amino acid length of the proteins under study, the highest numbers of mutations were located in the Env protein in 3 children. For children C127 and C137, most of the mutations were located in Nef.

\section{Discussion}

In mother-to-child-transmission pairs, at least half of the HLA alleles are shared between mother and child. This may have implications for 


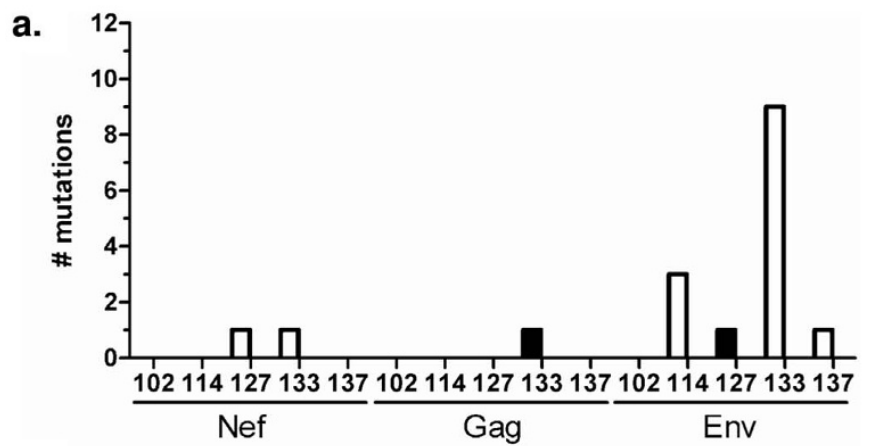

b.

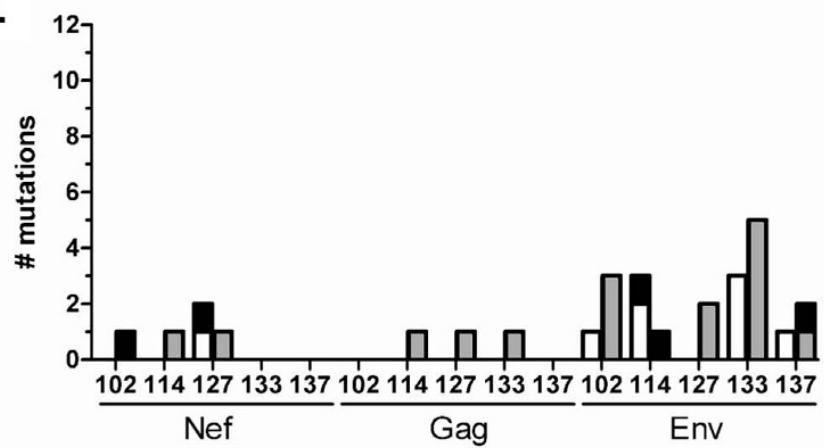

c.

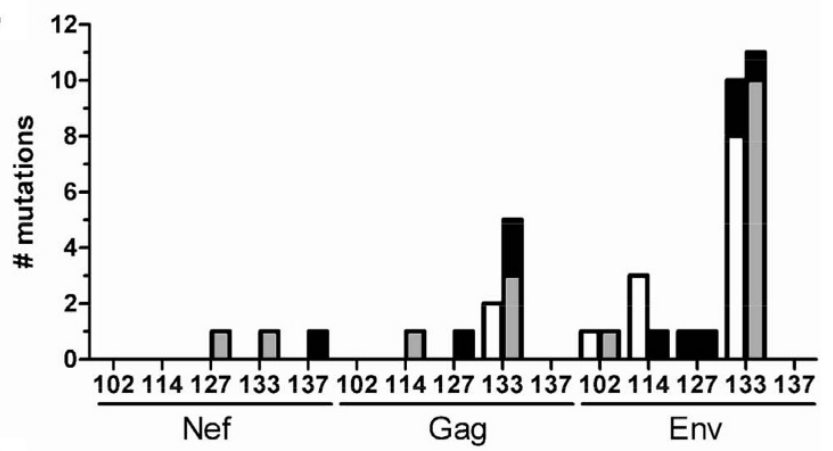

d.

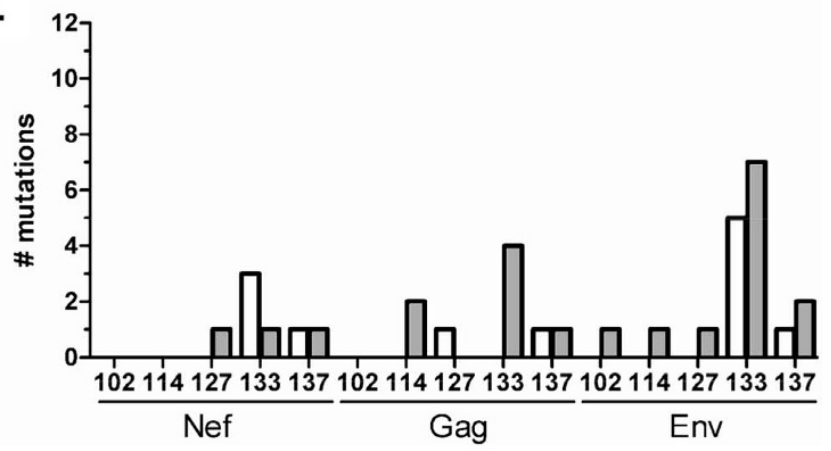

Figure $1 \mid$ Dynamics of CTL escape mutations after HIV-1 transmission from mother to child. Reversion of transmitted mutations (white) and forward mutations (grey) in HIV-1 variants obtained from the children. Mutations located at anchor residues of predicted CTL epitopes are depicted in black, both for reversions and forward mutations. (a). Reversion of transmitted mutations located in predicted CTL epitopes restricted by non-shared maternal HLA alleles. (b). Reversion of transmitted mutations and forward mutations located in predicted CTL epitopes restricted by HLA alleles that are shared between mother and child. (c). Reversion of transmitted mutations and forward mutations located in predicted CTL epitopes restricted by paternal HLA alleles from the child. (d). Reversion of transmitted mutations and forward mutations located outside of predicted CTL epitopes. the adaptive cellular immune response to HIV-1 in the infant, as CTL escape mutations in HIV-1 that developed in the mother may have been transmitted to the child. Here, we describe the viral evolution and the dynamics of CTL escape and reversion after transmission in the context of shared and non-shared HLA alleles in viral variants obtained from five mother-to-child transmission pairs.

The genetic divergence of sequences obtained from mother and child from time points close to delivery or birth, respectively, or from two successive time points of the children was around $2 \%$ for $n e f$ and gag, and around 3\% for env. The ratio of non-synonymous and synonymous mutations was calculated to determine whether the sequence divergence observed could be the result of selection pressure. Negative to neutral selection was mainly observed for Nef, Gag and Env gp160, with positive selection in the V1V2 and V4 variable regions of the viral envelope.

When analyzing the dynamics of CTL escape mutations and reversion of mutations after transmission, we observed large variation between the children. In one of the children studied (child C102), virtually no reversions and forward mutations were seen. As child C102 died 3.5 months after birth, an effective immune response may not have developed, thus explaining the extremely low evolution of the virus. Viral variants obtained from children C114, C127 and C137 displayed a higher evolution rate, whereas most evolution was observed in viral variants from child C133.

Inside predicted CTL epitopes restricted by non-shared maternal HLA alleles, the number of reversions of transmitted mutations in viral sequences was very low ( $0-3$ reversions) in 4 out of 5 children in all three viral genes under study. In sequences from child C133 more reversions of transmitted mutations were observed, with the majority located in Env.

Limited evolution was seen in CTL epitopes restricted by HLA alleles shared between mother and child. The low number of forward mutations in these epitopes suggests a relatively low selective pressure on epitopes restricted by shared HLA alleles, which may be the consequence of adaptation to immune pressure in the mother and subsequent transmission of adapted viral strains to the child. Furthermore, the limited number of reversions observed during the early phase of infection in the children may indicate that CTL escape mutations that developed in the mother did not come at a fitness cost or that the fitness cost has been compensated for before the transmission event. Within predicted epitopes restricted by HLA alleles from the children inherited from the father more evolution was observed. Viral evolution was dominated by forward mutations, which suggests CTL pressure on the presented epitopes leading to escape mutations. In line with this, child C133, who inherited HLA$B^{*} 2705$ from the father, displayed a relatively high number of forward mutations. HLA-B*2705 is associated with slower disease progression $^{30-33}$. Indeed, known HLA-B*2705 associated CTL escape mutations were observed in this child, and may account for the relatively high number of forward mutations. The highest number of mutations outside predicted CTL epitopes was also observed in child $\mathrm{C} 133$. These mutations might be compensatory mutations to restore viral fitness associated with escape from CTL pressure.

Although we only found limited viral evolution after mother-tochild transmission in our study, the observed viral evolution was dominated by forward mutations rather than reversion of transmitted mutations. This finding is surprising, since it has been reported that viral adaptation after horizontal transmission is mostly driven by reversions in the initial phase of HIV-1 infection ${ }^{34-36}$. However, a recent study on mother-to-child transmission pairs also reported a surprisingly low reversion rate of transmitted mutations ${ }^{37}$. These findings may indicate that the fitness cost of the transmitted mutations is low and therefore does not lead to reversion of these mutations. Alternatively, compensation of the fitness cost may occur by forward mutations in the child, or may have already occurred in the mother before transmission to the child. 
The majority of the observed mutations in viral variants obtained from the children were located in the Env protein, and positive selection was observed in the variable regions V1V2 (C114, C133 and $\mathrm{C} 137)$ and V4 (C102, C114 and C127). Evolution in these regions may be driven by the development of neutralizing antibodies. However, only a very limited antibody response against autologous viral variants was observed in serum from the children (data not shown). For mother-child pairs 114 and 133 we did observe a small increase in the sensitivity of viral variants from the children to antibodies in the serum of the mother (data not shown). This may suggest that evolution in the Env region in the children might be driven by changes to a more optimal conformation when antibody pressure is relieved, or may involve regions that play a role in receptor binding and target cell entry.

Although the level of viral evolution during the early phase after vertical transmission varied widely between the children, a limited number of mutations (both reversions and forward mutations) was observed overall in viral sequences obtained from the 5 children. This may indicate low selection pressure on the virus, which may be partially explained by viral adaptation to CTL pressure to epitopes restricted by HLA alleles that are shared between mother and child. It has been reported that children who acquired HIV-1 through mother-to-child transmission tend to have a worse prognosis than children who acquired infection through blood transfusion, suggesting that adaptation to shared HLA alleles influences the clinical outcome ${ }^{38}$.

CTL epitopes were predicted with the online prediction program Motif Scan on the Los Alamos Database website. This tool predicts the binding of epitopes in the binding pockets of HLA alleles. However, proteasomal cleavage and TAP transport are not taken into account with the Motif Scan tool. The cleavage and transport of viral proteins can be calculated with the online prediction program ImmuneEpitope (http://www.iedb.org) ${ }^{39}$. When this program was used for CTL epitope prediction, similar results on the dynamics of reversion and forward mutations in viral sequences from the children were obtained. However, not all the HLA alleles expressed by the mothers and children studied were included in the ImmuneEpitope program, therefore we chose to use the Motif Scan tool for our analysis. To make sure no described epitopes were missed in our analysis, known epitopes published in the HIV Molecular Immunology compendium 2009 were additionally analyzed. Published CTL epitopes are mainly composed of epitopes presented by either protective HLA alleles or HLA alleles best studied, and in a recent study by Ryland et al. ${ }^{37}$, a high degree of mismatch between viral sequences studied and the sequences published in epitope databases was observed, which suggests limitations for the sole use of published CTL epitopes to study evolution within epitopes targeted by CTLs. An important advantage of using CTL epitope prediction programs is the opportunity to study epitopes presented by HLA alleles that have not been extensively studied. However, prediction of viral epitopes that can be presented by HLA molecules does not give information about whether the predicted epitopes do in fact elicit CTL responses. In a paper by Schellens et al. most of the predicted epitopes tested elicited good IFN $\gamma$ responses in patient CTLs, suggesting that epitope prediction programs can be a good means to study evolution in CTL epitopes ${ }^{40}$.

Our sequence analysis was performed on clonal HIV-1 variants isolated from single productively infected cells as this allows the comparison of sequence dynamics in different genes of a single virus variant. One could argue that these viruses may not be fully representative of the total replication competent viral quasispecies in plasma, since the PBMC-associated viruses represent recent and archived viruses. However, recently we demonstrated that viral sequences from PBMC-DNA, PBMC clonal variants and serum belong to the same viral population and clonal variants may equally represent the viral quasispecies in blood in vivo ${ }^{41}$.
In conclusion, this study describes limited viral evolution in the early phase of HIV-1 infection after mother-to-child transmission. Viral genetic divergence was low in nef, gag and env in all children and mostly negative to neutral selection was observed. Positive selection pressure $(\mathrm{dN} / \mathrm{dS}>1)$ was observed in the V1V2 and V4 variable regions of Env. Furthermore, a limited number of reversions was observed inside CTL epitopes restricted by non-shared maternal HLA alleles and by HLA alleles that are shared by mother and child, which suggests CTL escape mutations that have been transmitted did not come at a fitness cost or that the fitness cost had already been compensated for in the mother. A low number of forward mutations was observed in epitopes restricted by shared HLA alleles, which is indicative of a low CTL pressure on these epitopes in the children. The highest numbers of reversions and forward mutations in viral sequences from these children were found within predicted CTL epitopes associated with HLA alleles inherited paternally, indicating that viral evolution in the children is at least in part driven by CTL responses restricted by paternal HLA alleles.

\section{Methods}

Study participants. Five mother-child pairs participating in a Dutch prospective study of HIV-seropositive women and their children, between whom vertical HIV-1 transmission was documented, were included ${ }^{28}$. The children were born between 1989 and 1991. None of the mothers received antiretroviral therapy during pregnancy, and the children were not breast fed.

Four mother-child pairs were infected with HIV-1 subtype B, the fifth pair was infected with HIV-1 circulating recombinant form AG, as was confirmed by phylogenetic analysis of nef, gag and env sequences.

This study was approved by the Academic Medical Center Amsterdam institutional medical ethics committee and written consent was obtained. Experiments were conducted in accordance with the ethical principles set out in the Declaration of Helsinki.

Isolation of clonal HIV-1 variants. Isolation of biological virus clones was performed as previously described ${ }^{42,43}$. Patient PMBC obtained from varying time points during pregnancy (mother) and after delivery (both mother and child) were co-cultivated with 3 day PHA-stimulated PBMC ( $1 \mu \mathrm{g} / \mathrm{ml}$ PHA) from healthy donors for 35 days. To avoid the loss of slowly replicating viral variants from the patients, we used a protocol in which limiting numbers of infected patient PBMCs and stimulated healthy donor PBMCs are mixed in multiple parallel cultures, which allows for the isolation of multiple replication competent clonal variants ${ }^{42,43}$. Culture supernatants were tested weekly for virus production in an in-house gag p24 antigen capture ELISA $^{57}$, and one-third of the culture volume was transferred to a new 96-well plate and fresh PHA-stimulated PBMC were added to propagate the culture. If less than one-third of the cultures were p24-positive, cultures were considered to be infected by progeny of a single HIV infected cell ${ }^{28}$ and these samples were used for DNA isolation. The advantage of this method is that only full-length replication competent viruses are isolated and sequenced, and that we know the exact gag-env-nef composition of the viruses.

DNA isolation, PCR amplification and sequencing. Total DNA was isolated from PBMC infected with clonal HIV-1 isolates using the modified L6 isolation $\operatorname{method}^{44,45}$. For the amplification of $g a g$, a nested PCR approach was performed using the following primer combinations: Gag-fw (5' -CGACGCAGGACTCGGCTTGCTG-3') and Gag-outer rev (5'-GCCTGTCTCTCAGTAC-3') followed by 2 different nested PCR primer sets, Gag-BssHII-fw (5' -TGCTGAAGCGCCCGCACGGC-3') in combination with Gag-P17-rev (5'-CAAAACTCTTGCCTTATGG-3') and Gag-P17-fw ( $5^{\prime}$-TGCTAAACACAGTGGGGGGACAT- $\left.3^{\prime}\right)$ in combination with Gag-ApaI-rev (5'-TTCCTAGGGGCCCTGCAA-3'). For the amplification of nef primers Nef-1-fw (5' -AGCCATAGCAGTAGCTGAGG-3') and Nef-1-rev (5' GCTTATATGCAGGATCTGAGG-3') were used. Env was PCR amplified using the following primer combinations: for the primary PCR primers EnvA-fw $\left(5^{\prime}\right.$-GGCTTAGGCATCTCCTATGGCAGGAAGAA-3') and Env3Rlong-rev (5'-GGTGTGTAGTTCTGCCAATCAGGGAAGWAGCCTTGTGTG-3') were used, the nested PCR was conducted with primers Env_2Flong-fw (5'-GGTTAATTGATAGAATWAGRGAAAGAGCAGAAGACAGTGGCAATG-3') and Nef5-rev (5' -CCCWTCCAGTCCCCCCTT TTCTTT TAAAAAG-3').

Env PCR products were gel purified using the QIAquick Gel Extraction Kit (Qiagen) and cloned into the pCR4-TOPO vector from the TOPO TA Cloning Kit for Sequencing (Invitrogen) according to the manufacturer's protocol. Next, transformation into One-shot TOP10 chemically competent E. coli was performed followed by culture in the presence of ampicillin. Gag and nef PCR products were purified with EXOSAP-IT (USB, Cleveland, Ohio, USA) and sequenced with the ABI prism Bid Dye Terminator v1.1 Cyclesequencing kit (Applied Biosystems) using the PCR primers Gag-BssHII-fw, Gag-P17-rev, Gag-P17-fw and Gag-ApaI-rev and Nef1 -fw and Nef-1-rev. Sequencing of env was performed on an Applied Biosystems 3730xl Genetic Analyzer using the following primers: M13F-fw (5'-GTAAAACG- 
ACGGCCAG-3'), M13R-rev (5'-CAGGAAACAGCTATGAC-3'), Senv5-fw (5' GGTACCTGTGTGGAAAGA-3'), F112-fw (5' -CAGTACAATGYACACATGGRAT-3') and EnvSeqF-fw (5'-TTCAGACCTGGAGGAGGARATATGA -3').

Phylogenetic analysis. Sequences of nef, gag and $e n v$ were aligned with ClustalW in the BioEdit package software (BioEdit v 7.0.5, Tom Hall, Ibis Therapeutics, Carlsbad, CA) ${ }^{46}$ and checked manually. Sequences were submitted to Genbank (accession numbers KJ669727-KJ670070). Neighbor joining phylogenetic trees were made under the Hasegawa-Kishino-Yano (HKY85) model of evolution ${ }^{47}$ with PAUP* 4.0 beta 8 software (http://paup.csit.fsu.edu). Phylogenetic confidence was assessed by bootstrap with 1000 replicates.

Genetic divergence and selection pressure. Genetic divergence between viral sequences generated from mothers and their children or from children generated at two successive time points were analyzed with the Kimura- 2 parameter model of evolution in the MEGA 4.1 software package ${ }^{48}$. Genetic selection pressure on the viruses was analyzed by determining the number of synonymous (dS) and nonsynonymous $(\mathrm{dN})$ mutations with the SNAP tool from the Los Alamos database website (http://www.hiv.lanl.gov) ${ }^{49}$. $\mathrm{dN} / \mathrm{dS}$ ratios were calculated comparing viral variants isolated from the mothers around the time of delivery and from their children, or when available, comparing viral variants from the children isolated from two successive time points.

Dynamics of forward mutations and reversions of transmitted mutations. The number of amino acid substitutions in the nef, gag and env regions of viral sequences from the children compared to viral sequences from the mothers before delivery were counted and the number of forward mutations and transmitted mutations that reverted in viral variants in the children were analyzed. Forward mutations were defined as amino acid mutations in viral sequences in the child that were not observed in the viral sequences from the mother before delivery. Reversion of mutations in viruses from the children were defined as amino acid mutations present in virus variants from the mother that were transmitted to the child and subsequent back mutation of this amino acid towards consensus sequence in viral variants from the child. Mutations were considered when present in at least $25 \%$ of the viral variants from the child from a single time point. The number of reversions and forward mutations were categorized as being outside or inside CTL epitopes restricted by HLA alleles from the mother that are not shared with the child (maternal), HLA alleles that both mother and child carry (shared), or HLA alleles from the child that are not shared with the mother (paternal).

When a mutation was located in more than 1 CTL epitope with the same HLA inheritance pattern (maternal non-shared, shared, paternal non-shared), the mutation was only counted once. Mutations located in more than 1 CTL epitope with different HLA inheritance patterns were categorized in both groups. For the prediction of potential CTL epitopes, the Motif Scan tool on the Los Alamos database website was used ${ }^{50-52}$. Four digit genotyping of HLA class I loci was performed for all mothers and children by sequence specific primers (SSP) PCR as described elsewhere ${ }^{53}$ and the HLA alleles expressed by mothers and children were used to predict CTL epitopes in the viral proteins with a length of 9 amino acids. Not all of the HLA alleles from our study participants were included in the Motif Scan tool. Therefore, custom motifs described in literature for HLA alleles $A^{*} 2301^{50}, A^{*} 7401^{54}, B^{*} 1801^{55}$ and $B^{*} 4501^{55}$ were used for prediction of CTL epitopes. In addition, the best-known HLA-restricted CTL epitopes published in the HIV Molecular Immunology compendium $2009^{56}$ were included in our analysis.

1. Koup, R. A. et al. Temporal association of cellular immune responses with the initial control of viremia in primary human immunodeficiency virus type 1 syndrome. J. Virol. 68, 4650-4655 (1994).

2. Borrow, P., Lewicki, H., Hahn, B. H., Shaw, G. M. \& Oldstone, M. B. A. Virusspecific CD8 + cytotoxic T-lymphocyte activity associated with control of viremia in primary human immunodeficiency virus type 1 infection. J. Virol. 68, 6103-6110 (1994).

3. Schmitz, J. E. et al. Control of viremia in simian immunodeficiency virus infection by CD8+ lymphocytes. Science 283, 857-860 (1999).

4. Streeck, H. et al. Human immunodeficiency virus type 1-specific CD8+ T-cell responses during primary infection are major determinants of the viral set point and loss of CD4+ T cells. J. Virol. 83, 7641-7648 (2009).

5. Friedrich, T. C. et al. Subdominant CD8+ T-cell responses are involved in durable control of AIDS virus replication. J. Virol. 81, 3465-3476 (2007).

6. Klein, M. R. et al. Kinetics of Gag-specific CTL responses during the clinical course of HIV-1 infection: A longitudinal analysis of rapid progressors and long-term asymptomatics. J. Exp. Med. 181, 1365-1372 (1995).

7. Jin, X. et al. Dramatic rise in plasma viremia after CD8(+) $\mathrm{T}$ cell depletion in simian immunodeficiency virus-infected macaques. J. Exp. Med. 189, 991-998 (1999).

8. Draenert, R. et al. Immune selection for altered antigen processing leads to cytotoxic T lymphocyte escape in chronic HIV-1 infection. J Exp Med 199, 905-915 (2004).

9. Allen, T. M. et al. Selection, transmission, and reversion of an antigen-processing cytotoxic T-lymphocyte escape mutation in human immunodeficiency virus type 1 infection. J Virol 78, 7069-7078 (2004).
10. Yokomaku, Y. et al. Impaired processing and presentation of cytotoxic-Tlymphocyte (CTL) epitopes are major escape mechanisms from CTL immune pressure in human immunodeficiency virus type 1 infection. J. Virol. 78, 1324-1332 (2004).

11. Goulder, P. J. et al. Late escape from an immunodominant cytotoxic Tlymphocyte response associated with progression to AIDS. Nat. Med. 3, 212-217 (1997).

12. Koenig, S. et al. Transfer of HIV-1 specific cytotoxic T lymphocytes to an AIDS patient leads to selection for mutant HIV variants and subsequent disease progression. Nature Medicine 1, 330-336 (1995).

13. Barouch, D. H. et al. Eventual AIDS vaccine failure in a rhesus monkey by viral escape from cytotoxic T lymphocytes. Nature 415, 335-339 (2002).

14. Navis, M. et al. Viral replication capacity as a correlate of HLA B57/B5801associated nonprogressive HIV-1 infection. J. Immunol. 179, 3133-3143 (2007).

15. Goepfert, P. A. et al. Transmission of HIV-1 Gag immune escape mutations is associated with reduced viral load in linked recipients. J. Exp. Med. 205, 1009-1017 (2008).

16. Peyerl, F. W. et al. Fitness costs limit viral escape from cytotoxic T lymphocytes at a structurally constrained epitope. J. Virol. 78, 13901-13910 (2004).

17. Leslie, A. J. et al. HIV evolution: CTL escape mutation and reversion after transmission. Nat. Med. 10, 282-289 (2004).

18. Friedrich, T. C. et al. Reversion of CTL escape-variant immunodeficiency viruses in vivo. Nat. Med. 10, 275-281 (2004).

19. Scott-Algara, D. et al. Not all tetramer binding CD8 + T cells can produce cytokines and chemokines involved in the effector functions of virus-specific CD8+ T lymphocytes in HIV-1 infected children. J. Clin. Immunol. 25, 57-67 (2005).

20. Pillay, T. \& Phillips, R. E. Adaptive evolution in perinatal HIV-1. Best. Pract. Res. Clin. Obstet. Gynaecol. 19, 211-229 (2005).

21. Thobakgale, C. F. et al. Human immunodeficiency virus-specific CD8+ T-cell activity is detectable from birth in the majority of in utero-infected infants. J. Virol. 81, 12775-12784 (2007).

22. Sanchez-Merino, V., Nie, S. \& Luzuriaga, K. HIV-1-specific CD8 + T cell responses and viral evolution in women and infants. J. Immunol. 175, 6976-6986 (2005).

23. Thobakgale, C. F. et al. Impact of HLA in mother and child on disease progression of pediatric human immunodeficiency virus type 1 infection. J. Virol. 83, 10234-10244 (2009).

24. Goulder, P. J. et al. Evolution and transmission of stable CTL escape mutations in HIV infection. Nature 412, 334-338 (2001).

25. Schneidewind, A. et al. Maternal transmission of human immunodeficiency virus escape mutations subverts HLA-B57 immunodominance but facilitates viral control in the haploidentical infant. J. Virol. 83, 8616-8627 (2009).

26. Pillay, T. et al. Unique acquisition of cytotoxic T-lymphocyte escape mutants in infant human immunodeficiency virus type 1 infection. J. Virol. 79, 12100-12105 (2005).

27. Feeney, M. E. et al. HIV-1 viral escape in infancy followed by emergence of a variant-specific CTL response. J. Immunol. 174, 7524-7530 (2005).

28. Van't Wout, A. B. et al. Macrophage-tropic variants initiate human immunodeficiency virus type 1 infection after sexual, parenteral, and vertical transmission. J. Clin. Invest. 94, 2060-2067 (1994).

29. Mulder-Kampinga, G. A. et al. Genomic human immunodeficiency virus type RNA variation in mother and child following intra-uterine virus transmission. J. Gen. Virol. 74, 1747-1756 (1993).

30. Gao, X. et al. AIDS restriction HLA allotypes target distinct intervals of HIV-1 pathogenesis. Nat. Med. 11, 1290-1292 (2005).

31. Carrington, M. \& O'Brien, S. J. The influence of HLA genotype on AIDS. Annu. Rev. Med. 54, 535-551 (2003).

32. Hendel, H. et al. New class I and II HLA alleles strongly associated with opposite patterns of progression to AIDS. J. Immunol. 162, 6942-6946 (1999).

33. Goulder, P. J. R. et al. Late escape from an immunodominant cytotoxic Tlymphocyte response associated with progression to AIDS. Nature Medicine 3, 212-217 (1997).

34. Li, B. et al. Rapid reversion of sequence polymorphisms dominates early human immunodeficiency virus type 1 evolution. J. Virol. 81, 193-201 (2007).

35. Matthews, P. C. et al. Central role of reverting mutations in HLA associations with human immunodeficiency virus set point. J. Virol. 82, 8548-8559 (2008).

36. Duda, A. et al. HLA-associated clinical progression correlates with epitope reversion rates in early human immunodeficiency virus infection. J. Virol. 83, 1228-1239 (2009).

37. Ryland, E. G., Tang, Y., Christie, C. D. \& Feeney, M. E. Sequence evolution of HIV1 following mother-to-child transmission. J. Virol. 84, 12437-12444 (2010).

38. Frederick, T., Mascola, L., Eller, A., O’Neil, L. \& Byers, B. Progression of human immunodeficiency virus disease among infants and children infected perinatally with human immunodeficiency virus or through neonatal blood transfusion. Los Angeles County Pediatric AIDS Consortium and the Los Angeles CountyUniversity of Southern California Medical Center and the University of Southern California School of Medicine. Pediatr. Infect. Dis. J. 13, 1091-1097 (1994).

39. Vita, R. et al. The immune epitope database 2.0. Nucleic Acids Res. 38, D854-D862 (2010). 
40. Schellens, I. M., Kesmir, C., Miedema, F., van, B. D. \& Borghans, J. A. An unanticipated lack of consensus cytotoxic T lymphocyte epitopes in HIV-1 databases: the contribution of prediction programs. AIDS 22, 33-37 (2008).

41. Edo-Matas, D. et al. Genetic composition of replication competent clonal HIV-1 variants isolated from peripheral blood mononuclear cells (PBMC), HIV-1 proviral DNA from PBMC and HIV-1 RNA in serum in the course of HIV-1 infection. Virology 405, 492-504 (2010).

42. Schuitemaker, H. et al. Biological phenotype of human immunodeficiency virus type 1 clones at different stages of infection: progression of disease is associated with a shift from monocytotropic to T-cell-tropic virus population. J. Virol. 66, 1354-1360 (1992)

43. Van't Wout, A. B., Schuitemaker, H. \& Kootstra, N. A. Isolation and propagation of HIV-1 on peripheral blood mononuclear cells. Nat. Protoc. 3, 363-370 (2008).

44. Boom, R. et al. A rapid and simple method for purification of nucleic acids. J. Clin. Microbiol. 28, 495-503 (1991).

45. Kootstra, N. A. \& Schuitemaker, H. Phenotype of HIV-1 lacking a functional nuclear localization signal in matrix protein of GAG and $\mathrm{Vpr}$ is comparable to wild type HIV-1 in primary macrophages. Virology 253, 170-180 (1999).

46. Hall, T. A. BioEdit: a user-friendly biological sequence alignment editor and analysis program for Windows 95/98/NT. Nucl. Acids. Symp. Ser. 41, 95-98 (1999)

47. Hasegawa, M., Kishino, H. \& Yano, T. Dating of the human-ape splitting by a molecular clock of mitochondrial DNA. J. Mol. Evol. 22, 160-174 (1985).

48. Tamura, K., Dudley, J., Nei, M. \& Kumar, S. MEGA4: Molecular Evolutionary Genetics Analysis (MEGA) software version 4.0. Mol. Biol. Evol. 24, 1596-1599 (2007).

49. Korber, B. Computational Analysis of HIV Molecular Sequences in HIV Signature and Sequence Variation Analysis. (eds. Rodrigo, A. G. \& Learn, G. H.) 55-72 (Kluwer Academic Publishers, Dordrecht, 2000).

50. Sette, A. \& Sidney, J. Nine major HLA class I supertypes account for the vast preponderance of HLA-A and -B polymorphism. Immunogenetics 50, 201-212 (1999).

51. Rammensee, H., Bachmann, J., Emmerich, N. P., Bachor, O. A. \& Stevanovic, S. SYFPEITHI: database for MHC ligands and peptide motifs. Immunogenetics $\mathbf{5 0}$, 213-219 (1999).

52. Marsh, S. G. E., Parham, P. \& Barber, L. D. The HLA Factsbook. (Academic Press, San Diego, 2000)

53. Cao, K. et al. Analysis of the frequencies of HLA-A, B, and C alleles and haplotypes in the five major ethnic groups of the United States reveals high levels of diversity in these loci and contrasting distribution patterns in these populations. Hum. Immunol 62, 1009-1030 (2001).

54. Nielsen, M. et al. NetMHCpan, a method for quantitative predictions of peptide binding to any HLA-A and -B locus protein of known sequence. PLoS. ONE. 2, e796 (2007).
55. Hillen, N. et al. Essential differences in ligand presentation and T cell epitope recognition among HLA molecules of the HLA-B44 supertype. Eur. J. Immunol. 38, 2993-3003 (2008)

56. HIV Molecular Immunology. 2009. Los Alamos National Laboratory, Theoretical Biology and Biophysics, New Mexico, LA-UR 06-0036.

57. Tersmette, M. et al. Detection and subtyping of HIV-1 isolates with a panel of characterized monoclonal antibodies to HIV-p24 gag. Virology 171, 149-155 (1989).

\section{Acknowledgments}

The Amsterdam Cohort Studies on HIV infection and AIDS, a collaboration between the Amsterdam Health Service, the Academic Medical Center of the University of Amsterdam, Sanquin Blood Supply Foundation, the University Medical Center Utrecht, and the Jan van Goyen Clinic are part of the Netherlands HIV Monitoring Foundation and financially supported by the Center for Infectious Disease Control of the Netherlands National Institute for Public Health and the Environment. We are indebted to all participants for their continuous participation in the study. This work was financially supported by the Aids Fonds Netherlands [grant 2008023]

\section{Author contributions}

E.F.G. generated and analyzed data and wrote the manuscript. A.C.v.N., A.T.P., E.J.B. and G.B.S.-J. generated data. N.A.K. and H.S. designed the study and N.A.K. wrote the manuscript.

\section{Additional information}

Accession Codes Genbank accession numbers: KJ669727-KJ670070.

Supplementary information accompanies this paper at http://www.nature.com/ scientificreports

Competing financial interests: The authors declare no competing financial interests.

How to cite this article: Gijsbers, E.F. et al. Low level of HIV-1 evolution after transmission from mother to child. Sci. Rep. 4, 5079; DOI:10.1038/srep05079 (2014).

This work is licensed under a Creative Commons Attribution-NonCommercialNoDerivs 3.0 Unported License. The images in this article are included in the article's Creative Commons license, unless indicated otherwise in the image credit; if the image is not included under the Creative Commons license, users will need to obtain permission from the license holder in order to reproduce the image. To view a copy of this license, visit http://creativecommons.org/licenses/by-nc-nd/3.0/ 